Supporting Information

\title{
Nanocapsule pH Regulator: Sustained Continuous Alkali Release from Thermosensitive Liposomes Reduces Acid Erosion
}

\author{
Jian Rong Chong, ${ }^{\dagger}$ Duc Long Le,$^{\dagger}$ Hirotaka Sato $^{*, \dagger}$ and Keitaro Sou ${ }^{*, \dagger, \dagger}$
}

†School of Mechanical \& Aerospace Engineering, Nanyang Technological University 50 Nanyang Avenue, 639798, Singapore

Research Institute for Science and Engineering, Waseda University, 3-4-1 Ohkubo, Shinjuku, Tokyo 169-8555, Japan

*Corresponding authors

Hirotaka Sato, hirosato@ntu.edu.sg

Keitaro Sou,soukei@aoni.waseda.jp 


\section{Theoretical encapsulation efficiency of Tris-Lipo and sample calculations}

The theoretical entrapment capacity of Tris-Lipo was estimated based on geometrical calculations, as described below.

The encapsulation efficiency is defined as

$$
E_{\text {efficiency }}=\frac{\text { Total encapsulated volume }}{\text { Total volume }}
$$

The inner aqueous volume $(V)$ of the Tris-Lipo (outer diameter, Zavg. $d$ nm; bilayer thickness, $r \mathrm{~nm}$ ) was calculated as

$$
V_{\text {Lipo }}=\frac{4}{3} \pi\left(\frac{\text { Zavg.d }}{2}-r\right)^{3}
$$

The number of the Tris-Lipo $(N)$ in the extruded solution was calculated as lipids in the bilayer membrane $(N)$ was calculated as

$$
N=\frac{A_{\text {lipids }}}{A_{\text {Lipo }}}
$$

where $A_{\text {Lipo }}$ is surface area of Tris-Lipo calculated as

$$
A_{\text {Lipo }}=4 \pi\left(\frac{\text { Zavg.d }}{2}\right)^{2}+4 \pi\left(\frac{\text { Zavg.d }}{2}-r\right)^{2}
$$

and $A_{\text {lipids }}$ is the summation of total average area of membrane where $A$ is Avogadro's number and $M w$ is the average molecular weight of membrane lipids.

The calculation was performed for vesicles of DPPC/SA/PEG-DSPE (9/1/0.06, molar ratio, average $M w=689$ ) with $193.8 \mathrm{~nm}$ (based on DLS analysis of Tris-Calcein lipo synthesised for the measurement of encapsulation efficiency test) for the outer diameter. The bilayer thickness was estimated as $5 \mathrm{~nm}(r=5)$ based on photographic analysis of the TEM imaging and average area of DPPC membrane lipid is $0.4 \mathrm{~nm}^{2}$ [S1]. The distance between bilayers was assumed as 0 in this calculation.

In the sample calculation below for the encapsulation efficiency test in the manuscript, $100 \mathrm{mg}$ of DPPC/SA/PEG-DSPE lipid was solubilized in $5 \mathrm{ml}$ of Tris-Calcein.

1. $A_{\text {lipids }}=\frac{100 \mathrm{mg}}{689 \mathrm{gmol}^{-1}} \times A \times 0.4 \mathrm{~nm}^{2}=3.495 * 10^{19} \mathrm{~nm}^{2}$

2. $A_{\text {Lipo }}=4 \pi\left(\frac{193.8 \mathrm{~nm}}{2}\right)^{2}+4 \pi\left(\frac{193.8 \mathrm{~nm}}{2}-5 \mathrm{~nm}\right)^{2}=2.241 * 10^{5} \mathrm{~nm}^{2}$

3. $N=\frac{A_{\text {lipids }}}{A_{\text {Lipo }}}=1.56 * 10^{14}$

4. $V_{\text {Lipo }}=\frac{4}{3} \pi\left(\frac{193.8 \mathrm{~nm}}{2}-5\right)^{3}=3.251 * 10^{6} \mathrm{~nm}^{3}$

5. Total encapsulated volume $=N * V_{\text {Lipo }}=5.071 * 10^{20} \mathrm{~nm}^{3}$

6. $E_{\text {efficiency }}=\frac{5.071 * 10^{20} \mathrm{~nm}^{3}}{5 \mathrm{~cm}^{3}} * 100 \%=10.14 \%$ 


\section{Effect of $\mathrm{pH}$ on payload release rate of Tris-Lipo and $\mathrm{pH}$ change}

\section{Experiment method for reacidification experiment}

DI water $(48 \mathrm{~mL})$ was prepared in a glass beaker and left in the $36.5^{\circ} \mathrm{C}$ thermostatic water bath and a $\mathrm{pH}$ electrode and temperature probe from a data-logging $\mathrm{pH}$ meter was inserted into the beaker to monitor and $\log$ temperature and $\mathrm{pH}$ changes. Parafilm was used to cover the top of the beaker. When the temperature of the solution has reached $36.5^{\circ} \mathrm{C}$ in equilibrium with the environment, the $\mathrm{pH}$ of the environment is adjusted to 4.0 with $1 \mathrm{M}$ lactic acid solution. Tris-Lipo dispersion $(2 \mathrm{~mL})$ was added to make up $50 \mathrm{~mL}$ of the lactic acid/ Tris-Lipo environment. Final $\mathrm{pH}$ adjustments were made to adjust the solution $\mathrm{pH}$ to 4.0 with $1 \mathrm{M}$ lactic acid solution. No HA samples were added to the solution, and samplings were taken at $30 \mathrm{~min}$ intervals as per the cargo release characterization procedures to determine the cargo release.

After the sampling at $3.5 \mathrm{~h}, 20 \mu \mathrm{L}$ of $1 \mathrm{M}$ lactic acid is added to the solution and lightly stirred to bring the $\mathrm{pH}$ down to 4.3 . Afterwards, the experiment is conducted as per usual procedures for the subsequent $2 \mathrm{~h}$ and terminated at $5.5 \mathrm{~h}$.
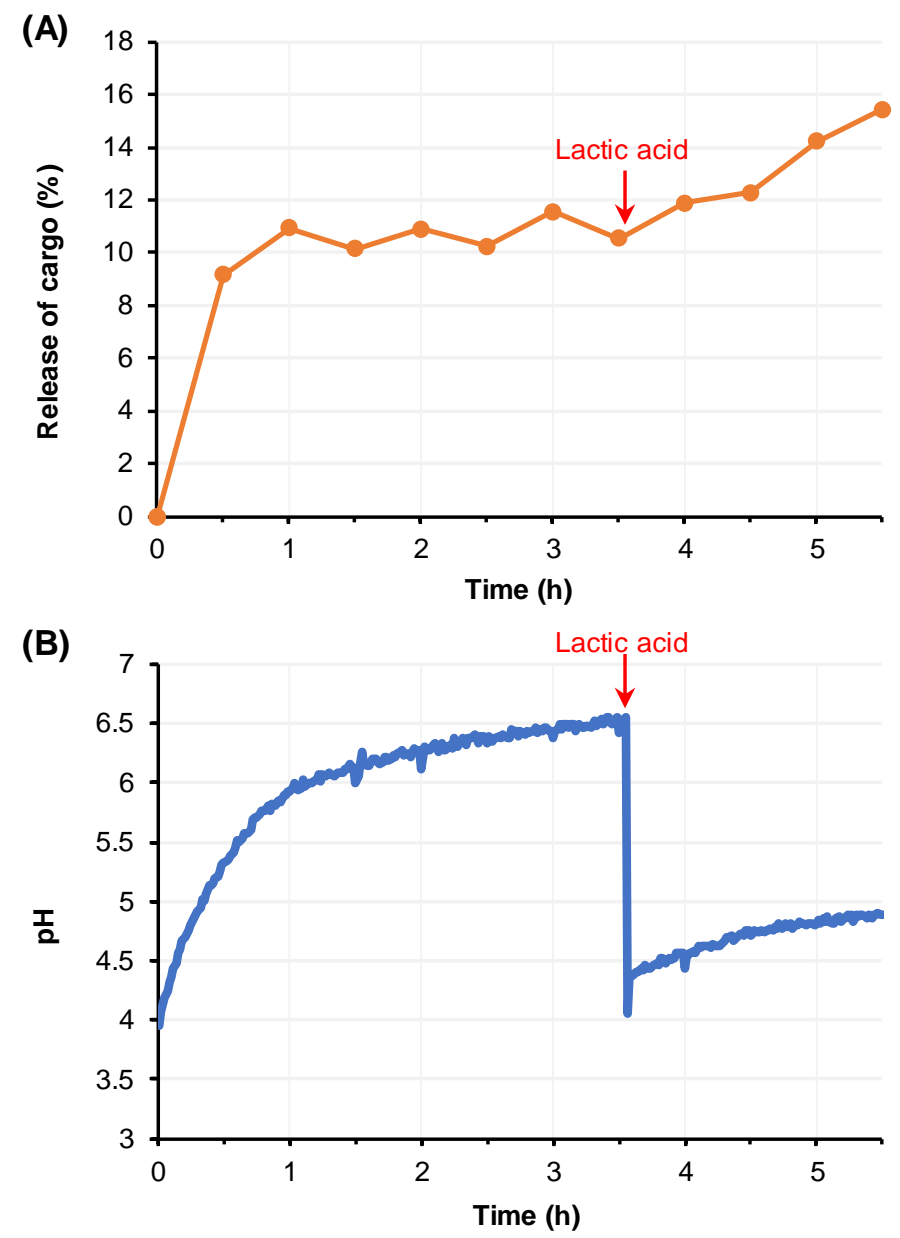

Figure S1. Profiles of (A) payload release and (B) $\mathrm{pH}$ change during incubation of TrisLipo dispersion at $36.5^{\circ} \mathrm{C}$. Lactic acid was added to the dispersion immediately after sampling at $3.5 \mathrm{~h}$. 
3. Erosion data of hydroxyapatite substrate (erosion and demineralization)

Table S1. Erosion data of Hydroxyapatite samples after 5 days of lactic acid immersion in-vitro

\begin{tabular}{|c|c|c|c|c|c|c|c|}
\hline Sample & $\begin{array}{c}\text { Reference } \\
\text { volume } \\
\left(\mathrm{mm}^{3}\right)\end{array}$ & $\begin{array}{l}\text { Volume } \\
\text { after } \\
\text { erosion } \\
\left(\mathrm{mm}^{3}\right)\end{array}$ & $\begin{array}{c}\text { Eroded } \\
\text { volume } \\
\left(\mathrm{mm}^{3}\right)\end{array}$ & $\begin{array}{c}\text { Average } \\
\text { eroded } \\
\text { volume } \\
\left(\mathrm{mm}^{3}\right)\end{array}$ & $\begin{array}{l}\text { Volume } \\
\text { after } \\
\text { sonication } \\
\left(\mathrm{mm}^{3}\right)\end{array}$ & $\begin{array}{c}\text { Demineralised } \\
\text { volume } \\
\left(\mathrm{mm}^{3}\right)\end{array}$ & $\begin{array}{c}\text { Average } \\
\text { demineralised } \\
\text { volume } \\
\left(\mathrm{mm}^{3}\right)\end{array}$ \\
\hline A1 & 0.179 & 0.319 & 0.14 & \multirow{5}{*}{$\begin{array}{c}0.187 \pm \\
0.05\end{array}$} & 0.348 & 0.029 & \multirow{5}{*}{$\begin{array}{c}0.078 \pm \\
0.04\end{array}$} \\
\hline A2 & 0.408 & 0.668 & 0.26 & & 0.706 & 0.038 & \\
\hline A3 & 0.801 & 0.944 & 0.143 & & 1.055 & 0.111 & \\
\hline A4 & 0.242 & 0.422 & 0.18 & & 0.540 & 0.118 & \\
\hline A5 & 0.397 & 0.611 & 0.214 & & 0.703 & 0.092 & \\
\hline L1 & 0.047 & 0.053 & 0.006 & \multirow{5}{*}{$\begin{array}{c}0.011 \pm \\
0.01\end{array}$} & 0.059 & 0.006 & \multirow{5}{*}{$0.018 \pm 0.01$} \\
\hline L2 & 0.045 & 0.048 & 0.003 & & 0.058 & 0.01 & \\
\hline L3 & 0.496 & 0.506 & 0.01 & & 0.543 & 0.037 & \\
\hline L4 & 0.089 & 0.09 & 0.001 & & 0.098 & 0.008 & \\
\hline L5 & 0.132 & 0.169 & 0.037 & & 0.196 & 0.027 & \\
\hline
\end{tabular}

\section{Reference}

[S1] Edholm, O.; Nagle, J. F., Areas of molecules in membranes consisting of mixtures. Biophys J. 2005, 89(3), 1827-1832. 